\title{
Background study of absorbed dose in biological experi- ments at the Modane Underground Laboratory
}

\author{
Nathanael Lampe,a, Pierre Marin ${ }^{1}$, Jean Castor $^{1}$, Guillaume Warot ${ }^{2}$, S. Incertit ${ }^{3,4}$, Lydia Maigne ${ }^{1,5}$, \\ David Sarramia ${ }^{1,5}$ and Vincent Breton ${ }^{1,5}$ \\ ${ }^{1}$ Université Clermont Auvergne, Université Blaise Pascal, CNRS/IN2P3, Laboratoire de Physique Corpusculaire, \\ BP 10448, F-63000 CLERMONT-FERRAND, France \\ ${ }^{2}$ Laboratoire Souterrain de Modane, 1125 Route de Bardonèche, 73500 MODANE, France \\ ${ }^{3}$ CENBG, UMR 5797, Université Bordeaux, F-33170 Gradignan, France \\ ${ }^{4} \mathrm{CNRS}$, IN2P3, UMR 5797, CENBG, F-33170, France \\ ${ }^{5}$ CNRS, IN2P3, UMR 6533, LPC, F-63178, France
}

\begin{abstract}
Aiming to explore how biological systems respond to ultra-low background environments, we report here our background studies for biological experiments in the Modane Underground Laboratory. We find that the minimum radioactive background for biology experiments is limited by the potassium content of the biological sample itself, coming from its nutritive medium, which we find in our experimental set-up to be $26 \mathrm{nGy} \mathrm{hr}^{-1}$. Compared to our reference radiation environment in Clermont-Ferrand, biological experiments can be conducted in the Modane laboratory with a radiation background 8.2 times lower than the reference above-ground level. As the radiation background may be further reduced by using different nutritive media, we also provide measurements of the potassium concentration by gamma spectroscopy of yeast extract $\left(63.3 \pm 1.2 \mathrm{mg} \mathrm{g}^{-1}\right)$ and tryptone $\left(2.5 \pm 0.2 \mathrm{mg} \mathrm{g}^{-1}\right)$ in order to guide media selection in future experiments.
\end{abstract}

\begin{abstract}
Afin d'examiner la manière avec laquelle les systèmes biologiques répondent aux environnements caractérisés par un bas niveau de radioactivité, nous avons mesuré le bruit de fond des expériences de biologie menées au Laboratoire Souterrain de Modane. Nous montrons que le bruit de fond est limité par le contenu en potassium au travers des échantillons biologiques euxmêmes, dans leurs milieux nutritifs. Dans le cadre des expériences examinées ici, le bruit de fond de potassium contribue à hauteur de $26 \mathrm{nGy} \mathrm{hr}^{-1}$. Par rapport à notre environnement de référence à Clermont-Ferrand, des expériences biologiques peuvent être conduites dans le laboratoire souterrain avec une réduction du bruit de fond de 8.2 fois le niveau de la surface. Du fait que le milieu nutritif apporte le plus grand impact au bruit de fond radiatif, nous recommandons la mesure par spectrométrie gamma de la concentration de potassium dans l'extrait de levure $\left(63.3 \pm 1.2 \mathrm{mg} \mathrm{g}^{-1}\right)$ et le tryptone $\left(2.5 \pm 0.2 \mathrm{mg} \mathrm{g}^{-1}\right)$ afin de guider la sélection du milieu des futures expériences.
\end{abstract}

\section{Introduction}

Underground laboratories provide a novel environment in which to conduct biological experiments, by offering a setting where the cosmic radiation flux is vastly reduced. Growing organisms inside this environment allows the contribution of the normal sea-level background dose received by cells to be suppressed, providing a means of exploring the impact of the natural radiative background on biological

\footnotetext{
${ }^{\mathrm{a}}$ corresponding author: lampe@clermont.in2p3.fr
} 
systems. Surprisingly, experiments led thus far in underground labs show that a reduction in background radiation has a stressful impact on cells, reducing the growth rate of bacteria when cells were grown in the Waste Isolation Pilot Plant in New Mexico [1], and reducing the ability of yeast cells grown in the Gran Sasso underground laboratory to withstand exposure to DNA damaging chemicals [2]. Both these works gave cells only a short time period to adapt to the underground environment ( $\sim 1$ week), and both indicate that the standard radiation background contributes to the maintenance or induction of mechanisms that either protect against cell damage from reactive oxygen species or repair damaged DNA. Low background experiments in Gran Sasso have been extended to study the impact of radiation on V79 Chinese hamster cells [3,4], and human lymphoblastoid TK6 cells [5]. Across the vast range of organisms considered, these experiments in underground laboratories all support the hypothesis that background radiation acts as a conditioning agent for the cellular response to DNA damage.

These works in underground laboratories align with a changing opinion in the literature that favours the idea that the biological response to radiation at low doses departs from a linear model [6], with authors suggesting that slightly increased levels of radiation may even be beneficial, according to the theory of radiation hormesis [7]. Conversely, current data does not provide a biological mechanism by which a low dose radiation threshold or hormesis effect could arise, nor evidence of a strong low dose threshold or strong radiation hormesis effect [8]. Regardless, as biology continues to explore this low radiation domain, accurate dose measurements become increasingly important as they, combined with measurements of DNA damage shed light on the exact nature of the low end of the dose-damage relationship.

The background radiation in underground laboratories comes from airborne radon and the decay of radioelements in the rock and concrete walls of the laboratory. A small amount of cosmic muons further penetrates the laboratory, and a thermal neutron flux is measurable coming from spontaneous fission of radioelements and $(\alpha, n)$ reactions in the surrounding rock [9]. The LSM (Laboratoire Souterrain de Modane/Modane Underground Laboratory) underground laboratory is situated on the French-Italian border along the Fréjus road tunnel, almost directly underneath the peak of the Fréjus Mountain. Here it is afforded a protection from cosmic radiation equivalent to $4,800 \mathrm{~m}$ of water [10].

The radiation background in the LSM is well documented. The radon level is maintained between 5 $\mathrm{Bq} \mathrm{m}^{-3}$ and $15 \mathrm{~Bq} \mathrm{~m}^{-3}$ [10]. Beyond the radon background, a gamma background is created from the decay chains of ${ }^{40} \mathrm{~K},{ }^{232} \mathrm{Th}$ and ${ }^{238} \mathrm{U}$ within the laboratory concrete and walls [11]. Measurements from [16] Malczewski et al. (2012), show the gamma ray flux in the LSM within the energy range 7.4-2734.2 $\mathrm{keV}$ to vary within $0.301-0.622 \gamma \mathrm{cm}^{-2} \mathrm{~s}^{-1}$ as one moves closer to the walls. Low numbers of cosmic muons penetrate into underground laboratories, with a vertical flux of $\phi_{\mu}=5.4 \pm$ $0.2 \mathrm{~m}^{-2}$ day $^{-1}$ [12], where they pose a problem for dark matter search experiments by creating secondary neutrons $[13,14]$. Thermal neutrons are also present in the LSM from spontaneous fission and $(\alpha, n)$ reactions, yielding $\phi_{n}=1.9 \times 10^{-6} \mathrm{~cm}^{-2} \mathrm{~s}^{-1}[15]$.

Biological experiments in underground labs also bring with them a level of radiation, in the form of ${ }^{40} \mathrm{~K}$ and ${ }^{14} \mathrm{C}$. Whilst this quantity varies between experiments, it along with the contributions to the radiative background from Radon and the telluric background, typically dominate the radiation dose received within a biological experiment.

In this paper, we present a summary of the biological set-up we will use within our experiments at Modane, followed by a summary of the methods we used to measure the absorbed dose within our experiment. We include in parallel measurements made at the LPC (Laboratoire de Physique Corpusculaire) in Clermont-Ferrand, to provide a measure of the dose reduction that it is possible to achieve in moving a biological experiment underground. Next we provide a summary of the dose measurements, coming from both direct measurements and simulation. The implications of these measurements are then discussed whilst we look at future perspectives for assessing DNA damage and methods of improving dose measurements. 


\section{Methods}

To provide a background to our method, it is important first to note some of the experimental conditions that these measurements are designed to support. Chiefly, the growth of the bacterium Escherichia coli B strain (REL606 and REL607) within a Davis minimal broth solution [16], within the low background environment provided by the LSM, both inside and outside lead shielding. Glucose is added to the solution with a concentration of $250 \mathrm{mg} \mathrm{L}^{-1}$. Similar experiments are also being conducted at the LPC, in order to provide a comparison point between normal and reduced background radiation levels. Accordingly, it has been important for us to measure the radiative background in the LSM and the LPC, as well as ascertain the contribution to the radiation dose in both these locations from the Davis minimal broth solution, which is rich in potassium and carbon.

Dose measurements made in the LSM were all taken within the recently added biology room with the detector in the centre of the room, or in cases noted as being "shielded" measurements, within a lead shield consisting of an interior layer of $5 \mathrm{~cm}$ of copper, surrounded by $10 \mathrm{~cm}$ of lead. Dose measurements of the background at the LPC were made in the microbiology laboratory attached to the LPC.

Measurements of the gamma spectra in each location were made using the $\mathrm{NaI}(\mathrm{Tl})$ detector of a handheld IdentiFinder Ultra-NGH. The detector chamber in the device is $36.0 \mathrm{~mm}$ in diameter and $50.8 \mathrm{~mm}$ long. Integration times varied for each observation varied from $7.46 \times 10^{4} \mathrm{~s}$ at the LPC to $2.61 \times 10^{3} \mathrm{~s}$ and $7.87 \times 10^{4} \mathrm{~s}$ in the unshielded and shielded LSM environments respectively. The measurements were made in Modane on March 18 and 19, 2015 and in Clermont-Ferrand from March 19-20.

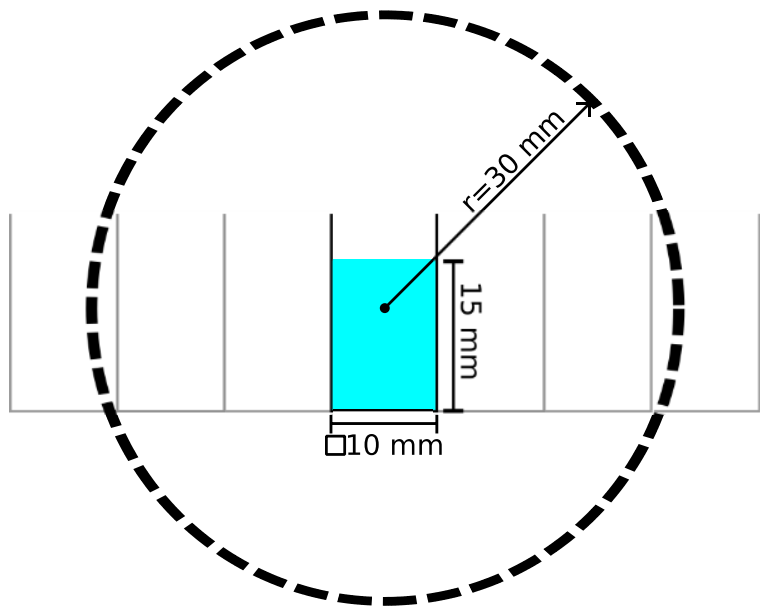

Figure 1. In Geant4, we simulate one well of a 96-well microplate filled with $1.5 \mathrm{~mL}$ of water as a detector. An isotropic background is simulated as a sphere of radius $30 \mathrm{~mm}$ around the well that emits particles following a cosine distribution. We also simulate the self-irradiation of the liquid by ${ }^{40} \mathrm{~K}$ and ${ }^{14} \mathrm{C}$. In this case, the source is the shaded detector region.

The gamma dose was measured using the dosimeter built into the IdentiFinder probe in each location. All dose measurements made were corroborated by secondary measurements from a Canberra Radiagem 2000 Personal Dose Rate and Survey Meter with a Very Low Dose Rate Probe attached. The ambient equivalent doses measured by these devices were converted to absorbed doses using the radiation weighting factor for gamma rays of 1 .

These doses were confirmed in simulation using the gamma spectra measured by Carlton [17], in order to validate the accuracy of these spectra as a simulation input. Using the Geant4 (version 10.01.patch01) simulation toolkit [18] we modelled our experimental geometry as a 96-well micro-plate with 25 wells 
filled with $1.5 \mathrm{~mL}$ of pure water, following the layout used in our experiment, which seeks to maximize the spacing between the 25 filled wells. Choosing one well towards the center, we measured the energy absorbed within this well when it was surrounded by an isotropic gamma source, emitting with the flux defined in

Table 1. Both the hall and wall sources were tested. The isotropic source was defined as a sphere of radius $3 \mathrm{~cm}$ around the centre of the well (Figure 1). Using each spectra, we simulated $10^{7}$ gamma rays, corresponding to 0.524 days for the spectrum at the wall and 1.08 days for the spectrum in the great hall. Physics was simulated using the "Livermore" physics constructor. The simulations were repeated using the default "StandardPhysics" constructor to ensure that results were consistent across different physics models. A secondary production cut of $1 \mu \mathrm{m}$ was used in both cases.

Table 1. Gamma fluxes in $\gamma \mathrm{cm}^{-2} \mathrm{~s}^{-1}$ measured by Malczewski [16] within the LSM, with the detector in near the centre of the main hall and flush against a wall.

\begin{tabular}{lllrrrc}
\hline Location & $7.4-249.8$ & $250.2-$ & $500.8-$ & $1005.6-$ & $1556.2-$ & $2056.2-$ \\
& $\mathrm{keV}$ & $500.4 \mathrm{keV}$ & $1005.2 \mathrm{keV}$ & $1555.8 \mathrm{keV}$ & $2055.8 \mathrm{keV}$ & $2734.2 \mathrm{keV}$ \\
\hline Hall & 0.185 & $5.3610^{-2}$ & $3.7410^{-2}$ & $1.8710^{-2}$ & $4.1010^{-3}$ & $2.4010^{-3}$ \\
Wall & 0.389 & 0.109 & $7.4710^{-2}$ & $3.5110^{-2}$ & $8.8910^{-3}$ & $5.0110^{-3}$ \\
\hline
\end{tabular}

The Davis minimal broth solution that provides nutrition for the bacteria in the experiment has an irradiative affect on the bacteria. We used simulation to measure the dose received from this source, based on the activity of the Davis medium solution found from its chemical composition. The DM250 solution we use (Davis medium without dextrose, sourced from Sigma-Aldrich product 15758 with $250 \mathrm{mg} \mathrm{L}^{-1}$ glucose added) carries a radioactivity from its potassium concentration of $115 \mathrm{~Bq} \mathrm{~L}^{-1}$. This activity was confirmed using a Germanium gamma spectrometer at the LPC Clermont-Ferrand. ${ }^{14} \mathrm{C}$ within the DM250, in it's standard abundance carries a minor contribution to the activity of $5.17 \times$ $10^{-2} \mathrm{~Bq} \mathrm{~L}^{-1}$. To simulate these sources, we kept the same simulation geometry as in Figure 1 except the water-filled well served as the particle source, for both beta electrons (in the cases of ${ }^{40} \mathrm{~K}$ and ${ }^{14} \mathrm{C}$ ) and for gamma rays $\left({ }^{40} \mathrm{~K}\right.$ only). For ${ }^{40} \mathrm{~K}, 10^{7}$ particles were simulated across two simulations. The first simulation measured the received dose from internal gamma emission, and the second from internal beta emission (we used the beta spectrum measured by Cameron \& Singh [19]. The $10^{7}$ primary particles simulated corresponded to simulation time periods of $6.16 \times 10^{3}$ days and $7.33 \times 10^{2}$ days respectively. For ${ }^{14} \mathrm{C}, 10^{7}$ electrons were simulated matching the element's beta spectrum (from Tilley [20]), corresponding to $1.49 \times 10^{7}$ days of experiment. Physics processes were modelled using the same methods as for the gamma background.

As part of providing a dose comparison between the LPC and LSM, it is necessary to estimate the radiation dose due to cosmic rays above ground at the LPC. The absorbed dose from charged cosmic rays and photons is reasonably uniform across most latitudes, and a good estimate is provided by the standard annual effective dose, which for these particles is identical to the absorbed dose [21]. Following Bouville \& Lowder [22], this value needs to be increased by $6 \%$ to account for the $400 \mathrm{~m}$ altitude of the LPC. The absorbed dose from neutrons is more difficult to calculate, and while standard effective dose numbers at sea level are available, the relationship between absorbed and effective neutron dose is a function of neutron energy. In order to estimate the absorbed dose in an experimental well above ground, we simulated the energy absorbed by a well in our experiment again using Geant4, keeping the same well geometry as in Figure 1 but with a modified source geometry. The modified source was a circular surface $150 \mathrm{~mm}$ in radius, $30 \mathrm{~mm}$ above the base of the well, centred directly above the well considered. Particles were generated along the down-facing side of this surface with their direction specified by an isotropic angular distribution. This is equivalent to simulating neutrons arriving at the well from an 
angle of $11.3^{\circ}$ above the horizon up to the zenith. The particles arrive at the well with an angular distribution of $\cos ^{2} \theta$ where $\theta$ is the angle from the zenith. $10^{7}$ particles were simulated, corresponding to 4.37 days of real time. The algebraic approximation of the neutron spectrum in New York [23] was used between $100 \mathrm{keV}$ and $500 \mathrm{MeV}$ in the simulation, giving a neutron flux of $\phi_{n}=5.96 \times 10^{-3} \mathrm{~cm}^{-2} \mathrm{~s}^{-1}$, and physics were modelled using the QGSP_BIC_HP physics list and a $1 \mathrm{~mm}$ secondary production cut.

Finally, in order to be able to compare the Davis Medium's activity to other commonly used biological media, we measured by gamma spectrometry the potassium concentrations of two common ingredients in biological experiments, yeast extract and tryptone. These are derived from biological processes rather than a mixture of chemicals. Yeast extract (Sigma-Aldrich 70161) is a nutrient reach medium containing autolysed yeast cells. We measured a $2.65 \mathrm{~g}$ sample of yeast extract for $190,450 \mathrm{~s}$. Tryptone (Sigma-Aldrich T9410) is a peptide mixture made from the digestion of casein by the tryptase enzyme. $2.77 \mathrm{~g}$ of tryptone was measured for $239,602 \mathrm{~s}$. In both measurements the potassium concentration was found from the size of the $1.46 \mathrm{MeV}$ gamma ray peak in the spectrum, and was corrected for the detector background, geometry and efficiency.

\section{Results.}

We present first our gamma spectrum measurements made using the $\mathrm{NaI}(\mathrm{Tl})$ detector (Figure 2). The absolute count rate at the LPC was significantly higher than the count rate in the LSM, at $10.57 \gamma \mathrm{s}^{-1}$ compared to $3.16 \gamma \mathrm{s}^{-1}$ and $0.092 \gamma^{-1}$ in the LSM biology room and lead shielding respectively. A steep drop is observed in the LSM biology room at counts above $1,650 \mathrm{keV}$ as the integration time was not sufficient to observe gamma rays at this energy. The curves for the LPC and shielded LSM environments do extend beyond this to $3,068 \mathrm{keV}$, however there are very few counts in this region (in both cases, $<0.6 \%$ of total counts). Across the entire range of energies sampled, the gamma background in Clermont-Ferrand remains approximately 100 times greater than that in the shielded LSM environment, and 3 times greater the unshielded environment.

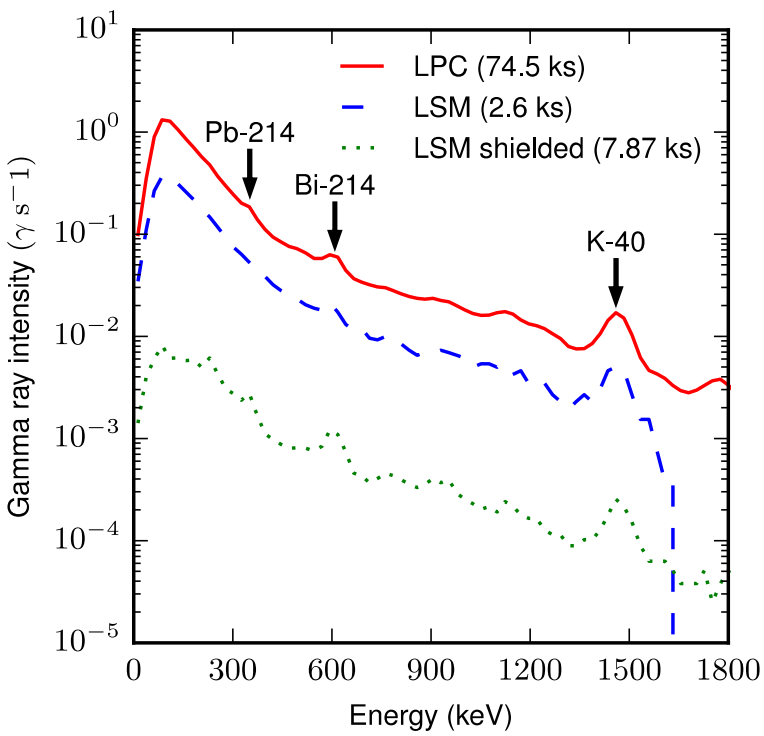

Figure 2. Comparison of gamma spectra at Clermont-Ferrand (LPC, solid red), the LSM biology lab (LSM, dashed blue), and inside the experimental lead chamber (LSM shielded, dotted green) measured with a NaI (Tl) detector. 
Whilst this measurement lacks the spectral resolution of a high purity germanium crystal, gamma peaks corresponding to some elements are evident and have been marked on Figure 2. These are all traceable to the primordial radionuclides that surround the laboratory. Most notable is the $1460 \mathrm{keV}$ peak from ${ }^{40} \mathrm{~K}$, and the $609.3 \mathrm{keV}$ peak from ${ }^{214} \mathrm{Bi}$, a radon daughter. An excess is observed in the curves near $338-362 \mathrm{keV}$, which is likely due to the $352.0 \mathrm{keV}$ peak of ${ }^{214} \mathrm{~Pb}$ (also a radon daughter).

Gamma dosimetry reveals the significant decrease in the gamma dose achieved by conducting experiments in the underground lab. Using the IdentiFinder gamma dosimeter, the gamma dose in the LSM biology room was found to be $20 \pm 5 \mathrm{nSv} \mathrm{hr}{ }^{-1}$, whilst in the Clermont-Ferrand microbiology laboratory the gamma dose was significantly higher, at $150 \pm 10 \mathrm{nSv} \mathrm{hr}{ }^{-1}$. Measurements made inside the lead chamber with the door ajar were not possible as the dose rate was below the detection threshold of the dosimeter. The IdentiFinder detector was limited to a precision of $10 \mathrm{nSv} \mathrm{hr}{ }^{-1}$.

Simulations of the energy deposited in water by the gamma background were made using energybinned flux measurements taken at a wall and in the centre of the LSM great hall. The results of these simulations are shown in Table 2. Both physics constructors used gave results in agreement with each other. The absorbed dose measured using the spectrum from the great hall is within the precision of that measured by dosimetry, whilst the spectrum against wall the overestimates the measured dose.

Table 2. Simulated absorbed dose rates from the gamma background in the LSM and the radiation sources in the nutritive medium. Doses were calculated using the Livermore and StandardPhysics constructors within Geant4. The gamma background was simulated using spectrum measured against a wall, and towards the centre of the laboratory hall.

\begin{tabular}{ccc}
\hline Source & $\begin{array}{c}\text { Livermore } \\
\left(\mathrm{nGy} \mathrm{hr}^{-1}\right)\end{array}$ & $\begin{array}{c}\text { Standard } \\
\left(\mathrm{nGy} \mathrm{hr}^{-1}\right)\end{array}$ \\
\hline Gamma (wall) & $47.3(4)$ & $47.9(4)$ \\
Gamma (hall) & $23.3(2)$ & $23.3(2)$ \\
${ }^{40} \mathrm{~K}(\gamma)$ & $25.865(5)$ & $25.939(5)$ \\
${ }^{40} \mathrm{~K}(\beta)$ & $0.1080(3)$ & $0.1088(3)$ \\
${ }^{14} \mathrm{C}(\beta)$ & $1.4757(3) 10^{-3}$ & $1.4762(3) 10^{-3}$ \\
\hline
\end{tabular}

The major internal contributions to the radiation dose were also simulated (Table 2). Firstly, the ${ }^{40} \mathrm{~K}$ gamma line has a very minor impact on radiation dose, and the radioactive decay of ${ }^{14} \mathrm{C}$ is negligible. Rather, the internal dose is dominated by the emission of beta particles from ${ }^{40} \mathrm{~K}$. It bears noting that the average energy of a ${ }^{40} \mathrm{~K}$ beta particle is $560 \mathrm{keV}$, however as electrons may escape the simulated well, an average of $426 \mathrm{keV}$ is deposited in the well per event. The energy that escapes may interact with another well. Whilst this depends strongly on the position of the well on the microplate (as not all wells have the same number of neighbouring wells), an approximation of the radiation dose in one well from another can be made by considering the angular space occupied by a neighbouring well. By considering the maximum solid angle that a neighbour may occupy and also assuming that little energy is lost in the air between wells, an upper bound can be found for the energy deposited in each neighbouring well per event $6.1 \mathrm{keV}$. That is, the dose is underestimated by $1.4 \%$ per neighbouring well. Thus, considering the geometry of a well in the centre of the microplate with six neighbours, the size of the underestimation in the ${ }^{40} \mathrm{~K}$ beta dose is no larger than $7 \%$.

The summed doses for each environment are presented in Table 3, where we have also marked the estimated neutron dose above ground $\left(4.4 \pm 0.3 \mathrm{nGy} \mathrm{hr}^{-1}\right)$ from simulation. The presence of internal ${ }^{40} \mathrm{~K}$ sets a limit upon the maximum dose reduction achievable following a protocol that uses Davis Medium. Merely conducting experiments in an underground laboratory reduces by a factor of 4.6 the background radiation level compared to the LPC. Removing the gamma background from the LSM using shielding allows a further reduction, such that the dose compared to the LPC is reduced by a factor of 8.2 .

In order to illustrate how other biological media could be used to reduce the internal emission of ionising radiation, we show in Table 3 the concentration of potassium in various biological powders, 
and the associated activity of ${ }^{40} \mathrm{~K}$. In both yeast extract and tryptone, there is significantly less potassium than in Davis Medium powder. Whilst the potassium concentration of a biological culture depends on the levels of yeast extract and tryptone demanded by the recipe, large reductions in concentration can be made by using media other than the Davis Medium. For example, 1 L of Lysogeny Broth (containing $10 \mathrm{~g} \mathrm{~L}^{-1}$ tryptone, $5 \mathrm{~g} \mathrm{~L}^{-1}$ yeast extract) contains $341.5 \mathrm{mg} \mathrm{L}^{-1}$ of potassium, a reduction in the potassium concentration by a factor of 10.9 times from Davis Medium (containing $10.6 \mathrm{~g} \mathrm{~L}^{-1}$ powder typically).

Table 3. Doses relevant to biological experiments at the LSM, relative to measurements made at the LPC Clermont-Ferrand. Dosimetry measurements exhibited a time variation on the order of $20 \%$ on both sides of the value cited. The background for cosmic rays was taken from UNSCEAR [24]. Doses from potassium and carbon inside the well were simulated using the Geant4 simulation toolkit.

\begin{tabular}{ll|ccc}
\hline Source & Method & $\begin{array}{c}\text { LPC Clermont } \\
\left(\mathrm{nGy} \mathrm{hr}^{-1}\right)\end{array}$ & $\begin{array}{c}\text { LSM } \\
\left(\mathrm{nGy} \mathrm{hr}^{-1}\right)\end{array}$ & $\begin{array}{c}\text { LSM (shielded) } \\
\left(\mathrm{nGy} \mathrm{hr}^{-1}\right)\end{array}$ \\
\hline $\begin{array}{l}\boldsymbol{\gamma} \text { back- } \\
\text { ground }\end{array}$ & Dosimeter & 150 & 20 & $<1$ \\
$\begin{array}{l}\text { Cosmic rays } \\
\text { (charged \& }\end{array}$ & UNSCEAR & 33 & $<<1$ & $<<1$ \\
$\boldsymbol{\gamma})$ & Simulation & 4.4 & $<<1$ & \\
$\begin{array}{l}\text { Cosmic rays } \\
(\text { neutrons })\end{array}$ & Simulation & 0.13 & 0.13 & $<<1$ \\
${ }^{40} \mathrm{~K}(\boldsymbol{\gamma})$ & Simulation & 26 & 26 & 0.13 \\
${ }^{40} \mathrm{~K}(\boldsymbol{\beta})$ & Simulation & $<<1$ & $<<1$ & 26 \\
${ }^{14} \mathrm{C}(\boldsymbol{\beta})$ & Total & 214 & 46 & 26 \\
\hline & & & $<1$
\end{tabular}

Table 4. Measured concentrations, c, of potassium and activity, A, of $40 \mathrm{~K}$ within one kilogram of standard biological powders from gamma spectroscopy.

The measurement from Davis medium is provided for reference calculated from its chemical composition.

\begin{tabular}{l|cc}
\hline Medium (as powder) & $\mathrm{c}(\mathrm{K})\left(\mathrm{mg} \mathrm{g}^{-1}\right)$ & $\mathrm{A}\left({ }^{40} \mathrm{~K}\right)\left(\mathrm{Bq} \mathrm{kg}^{-1}\right)$ \\
\hline Yeast Extract & $63.3 \pm 1.2$ & $1,960 \pm 40$ \\
Tryptone & $2.5 \pm 0.2$ & $7.7 \pm 0.6$ \\
Davis Medium & 351 & 10,840 \\
\hline
\end{tabular}

\section{Discussion.}

Our results show that Modane provides an excellent environment to conduct low background biological experiments. Biological experiments introduce a unique nuance into the standard way of running a low background experiment, where typically it is necessary to eliminate as many background sources as possible, to as large a degree as possible. Practically, biological experiments bring with them a level of radioactivity in their natural chemical composition, by virtue of the important biological role of potassium. Thus for the majority of low background biological experiments, it is sufficient to reduce the background sufficiently that it is dominated by the internal contribution of ${ }^{40} \mathrm{~K}$. Methods of reducing the potassium dose in biological experiments have not been widely discussed, indeed some experiments have forgotten to include the contribution of potassium within growth media. Media selection plays an important role in reducing the potassium background. We have presented measurements of the potassium concentration of one manufacturer's yeast extract and tryptone. These measurements can serve as a guide to assessing the radiative impact of different media. Indeed, there is a ten-fold decrease in the potassium concentration, and thus ${ }^{40} \mathrm{~K}$ activity going from Davis Medium to Lysogeny Broth. Whilst this decrease is large, it still does not alter our conclusion that potassium is the dominant source of radiation dose in shielded underground biological experiments. If further reductions were necessary, or 
an experiment required a potassium rich medium, potassium enriched with ${ }^{39} \mathrm{~K}$ could be used to significantly reduce the radiation background.

Working in underground laboratories, the major significant external radiation source is the gamma background. This gamma background can be effectively suppressed using standard lead shielding. While above ground, lead shielding can reduce the gamma background, underground laboratories shelter biological experiments from the far more difficult to eliminate cosmic sources of radiation, typically neutrons and muons. This is responsible for a reduction in the absorbed dose by $35 \mathrm{nGy} \mathrm{hr}^{-1}$, according to our quantification of the neutron dose, and the standard estimate of the cosmic background dose from charged particles and photons. A higher reduction is often achievable given that most underground laboratories include other measures to reduce the background radiation level, such as low radioactivity concrete.

It should be noted that our measurement of the gamma background at the LPC are significantly than the standard terrestrial gamma background of $60 \mathrm{nGy} \mathrm{hr}^{-1}$ [21]. Whilst the measurement we made does convolute the terrestrial and cosmic gamma backgrounds, as the gamma dosimeter could not distinguish the two, this is not a significant factor in the large background measurement as the cosmic background dose is dominated by muon interactions. Rather, the LPC is located in the 'Massif Central' region of France, which is known to have a particularly high background radiation level.

We have not specifically considered ${ }^{222} \mathrm{Rn}$ and its daughters as an important contributor to the internal dose in these measurements. Whilst the energy contained in $\alpha$-particles from decaying ${ }^{222} \mathrm{Rn}$ is large, and can significantly impact a biological system, the experimental geometry can play a large role in varying this dose. Lungs, or a lung like system for example will receive a significantly larger radiation dose from radon, due to the frequent gas exchange, than a glass test tube containing bacteria in a nutritive medium, and little air. Even without elaborate measures to limit exposure, the impact of ${ }^{222} \mathrm{Rn}$ is relatively small, for example, in the Gran Sasso laboratory, [13] Fratini et al. (2015) find its contribution to be less than $1 \%$ of that of ${ }^{40} \mathrm{~K}\left(0.17 \mathrm{nGy} \mathrm{hr}^{-1}\right.$ in a $5 \mathrm{~Bq} \mathrm{~m}^{-3}$ environment $)$.

Our simulation measurements of the gamma background in the main hall are in agreement with gamma dosimetry measurements, and as such would serve as an appropriate input for future simulations of the gamma background in Modane. Conversely, the gamma spectrum taken next to the wall overestimates the gamma dose, which is unsurprising given that the geometry of the measurement is heavily directional. Whilst using an energy binned spectrum as a simulation input causes the spectrum to lose the distintive peaks that characterise a gamma spectrum, this has little effect on the underlying result as the majority of counts are in the continuum part of the spectrum.

Assessing the biological impact of a background reduction from the $214 \mathrm{nGy} \mathrm{hr}^{-1}$ an organism would receive on the surface in Clermont-Ferrand with the much lower $26 \mathrm{nGy} \mathrm{hr}^{-1}$ from an underground laboratory is difficult due to the unknown response of living systems in ultra-low background environments. Daly [25] suggests that double strand breaks (DSBs) from radiation exposure typically occur with a frequency of $\sim 0.004 \mathrm{DSB} \mathrm{Gy}^{-1} \mathrm{Mbp}^{-1}$. If a linear model for induced radiation damage with dose holds true, then there would be an 8-fold difference in the number of DSBs occuring within organisms outside an underground laboratory, compared to those studied within it. Following this logic, cells grown underground would be considerably less damaged than those grown above ground. Whilst this may be technically true, low background experiments up to now indicate an up regulation of the oxidative stress response in low background environments. As a more coherent picture emerges of organic behaviour in low dose environments, it is important that measurements of dose in experimental systems are accurately made, to enable an accurate comparison between the different low background radiation environments and experimental procedures.

\section{Conclusion.}

We have presented measurements of the radiation background for a biological experiment in the Modane Underground Laboratory. The primary conclusion from these measurements is that while most forms of radiation may be suppressed to allow the study of life in the absence of ionising radiation, 
living systems are innately dependant upon potassium, and the quantity of ${ }^{40} \mathrm{~K}$ within the biological experiment sets the minimum radiation dose that can reasonably be achieved. Our measurements and simulations show that when an experiment is shielded and an experimental configuration that minimises radon exposure is used, the most significant factor in the absorbed radiation dose of the sample is that due to potassium.

\section{Acknowledgements.}

The authors would like to acknowledge the support of the staff of the LSM, who provided wonderful technical support throughout the measurements conducted there. We also thank Didier Miallier for his aid with gamma spectroscopy. NL acknowledges the financial support of the Conseil General de l'Allier.

\section{References.}

1. H. Castillo et al., Stress induction in the bacteria Shewanella oneidensis and Deinococcus radiodurans in response to below-background ionizing radiation, International Journal of Radiation Biology, 3002(October), 1-33 (2015)

2. L. Satta, et al., Low environmental radiation background impairs biological defence of the yeast Saccharomyces cerevisiae to chemical radiomimetic agents, Mutation research, 347(3-4), 129-133 (1995)

3. L. Satta et al., Influence of a low background radiation environment on biochemical and biological responses in V79 cells, Radiation and environmental biophysics, 41(3), 217-224 (2002)

4. E. Fratini et al., Low-radiation environment affects the development of protection mechanisms in V79 cells. Radiation and Environmental Biophysics, 54(2), 183-194 (2015)

5. M.C. Carbone et al., The Cosmic Silence experiment: on the putative adaptive role of environmental ionizing radiation, Radiation and Environmental Biophysics, 48(2), 189-196. (2009)

6. M. Tubiana, L.E. Feinendegen \& J.M. Kaminski, The Linear No-Threshold Relationship Is Inconsistent with Radiation Biologic and Experimental Data, Radiology, 251(1) (2009)

7. L.E. Feinendegen, Evidence for beneficial low level radiation effects and radiation hormesis, British Journal of Radiology, 78(925), 3-7 (2005)

8. M.P. Little et al., Risks associated with low doses and low dose rates of ionizing radiation: why linearity may be (almost) the best we can do, Radiology, 251(1), 6-12 (2009)

9. A. Bettini, New underground laboratories: Europe, Asia and the Americas, Physics of the Dark Universe, 4, 36-40 (2014)

10. F. Piquemal, Modane underground laboratory: Status and project, The European Physical Journal Plus, 127(9), 110 (2012)

11. V. Chazal et al., Neutron background measurements in the Underground Laboratory of Modane, Astroparticle Physics, 9(2), 163-172 (1998)

12. B. Schmidt et al.,. Muon-induced background in the EDELWEISS dark matter search, Astroparticle Physics, 44, 28-39 (2013)

13. E. Armengaud et al., Background studies for the EDELWEISS dark matter experiment, Astroparticle Physics, 47, 1-9 (2013)

14. S. Fiorucci et al., Identification of backgrounds in the EDELWEISS-I dark matter search experiment, Astroparticle Physics, 28(1), 143-153. (2007) 
15. I. Savvidis et al., Underground low flux neutron background measurements in LSM using a large volume $(1 \mathrm{~m} 3)$ spherical proportional counter, Journal of Physics: Conference Series, 203, p.012030. (2010)

16. B.C. Carlton, \& B.J. Brown, Gene mutation. In P. Gerhardt, ed. Manual of methods for general bacteriology, Washington D.C.: American Society for Microbiology, pp. 222-242 (1981)

17. D. Malczewski, J. Kisiel \& J. Dorda, Gamma background measurements in the Laboratoire Souterrain de Modane, Journal of Radioanalytical and Nuclear Chemistry, 292(2), 751-756. (2012)

18. J. Allison et al., Geant4 developments and applications. IEEE Transactions on Nuclear Science, 53(1), 270-278 (2006)

19. J.A. Cameron \& B. Singh, Nuclear Data Sheets for A=40, Nuclear Data Sheets, 102(2), 293-513 (2004)

20. D.R. Tilley et al., Energy Levels of Light Nuclei, Revised Manuscript, 40441(November) (2012)

21. UNSCEAR, 2000. Annex B Exposures from natural radiation sources, United Nations Scientific Comittee on the Effects of Atomic Radiation, Annex B.

22. A.Bouville \& W.M. Lowder, Human Population Exposure to Cosmic Radiation. Radiat Prot Dosimetry, 24(1-4), 293-299 (1988)

23. M.S. Gordon et al., Measurement of the flux and energy spectrum of cosmic-ray induced neutrons on the ground, IEEE Transactions on Nuclear Science, 51(6), 3427-3434 (2004)

24. UNSCEAR, Sources and effects of ionizing radiation: Exposures of the public and workers from various sources of radiation (Vienna: United Nations Scientific Comittee on the Effects of Atomic Radiation, 2008).

25. M.J. Daly, Death by protein damage in irradiated cells, DNA Repair, 11(1), 12-21 (2012) 